\title{
Diffusion Limited Supercritical Water Oxidation (SCWO) in Microgravity Environments
}

\author{
M. C. Hicks, R. W. Lauver \\ NASA Glenn Research Center \\ U. G. Hegde \\ National Center for Space Exploration Research \\ T.J. Sikora \\ ZIN Technologies
}

\begin{abstract}
Tests designed to quantify the gravitational effects on thermal mixing and reactant injection in a Supercritical Water Oxidation (SCWO) reactor have recently been performed in the Zero Gravity Facility (ZGF) at NASA's Glenn Research Center. An artificial waste stream, comprising aqueous mixtures of methanol, was pressurized to approximately $250 \mathrm{~atm}$ and then heated to $450^{\circ} \mathrm{C}$. After uniform temperatures in the reactor were verified, a controlled injection of air was initiated through a specially designed injector to simulate diffusion limited reactions typical in most continuous flow reactors. Results from a thermal mapping of the reaction zone in both $1-\mathrm{g}$ and $0-\mathrm{g}$ environments are compared. Additionally, results of a numerical model of the test configuration are presented to illustrate first order effects on reactant mixing and thermal transport in the absence of gravity.
\end{abstract}

\section{INTRODUCTION}

Supercritical water oxidation ("SCWO") technologies provide a means for efficiently reducing liquid and/or combined liquid/solid waste streams into its constituent elements. Oxidation takes place at temperatures and pressures above the critical point of water (i.e., $374^{\circ} \mathrm{C}$ and $218 \mathrm{~atm}$ ) completely converting all hydrocarbon wastes into $\mathrm{H}_{2} \mathrm{O}$ and $\mathrm{CO}_{2}$. SCWO has become an attractive candidate technology for processing solid and liquid wastes for long duration space and extraterrestrial planetary missions because (i) required pre-processing of waste is minimal, (ii) product streams are benign, microbially inert, and easily reclaimed, (iii) waste conversion is complete and relatively fast, and (iv) with proper design and operation reactions can be self-sustaining.

The extent to which reactions in a SCWO reactor will be impacted by the absence of gravitationally induced convection is an area that has yet to be studied. It is well established that diffusive flame systems, in the absence of gravity, show significant changes in both reaction rates and flame geometries. This is largely because reactant and thermal transport in these systems is often dominated by thermal and mass diffusion to the reaction zone rather than convective transport. Since gravitationally induced mixing is often the primary motive force of well mixed systems in many SCWO reactor designs, its absence or alteration will have significant implications in the design and/or operation of this technology in any future space/extraterrestrial application.

The unique qualities of water at supercritical temperatures and pressures result in profound changes in transport properties, thermal and caloric state variables, and solvating properties allowing for high reaction rates and conversion efficiencies over a wide range of waste streams. In particular, these changes allow for the oxidation of hydrocarbons to proceed at rates often approaching those found in gas combustion systems with conversion efficiencies typically above $99.99 \%$.

Figure 1 provides an illustration of the changes in the thermophysical properties of pure water as a function of temperature at a pressure of 250 bar. In this plot the values for density, thermal conductivity, viscosity and specific heat are normalized by their respective values at a reference state taken at $0^{\circ} \mathrm{C}$. It is observed that, within a relatively narrow transitional region (i.e., temperatures within $25^{\circ} \mathrm{C}$ of the critical temperature), the density decreases by nearly $80 \%$, the thermal conductivity decreases by $70 \%$, and the specific heat exhibits an interesting singularity, rapidly rising and falling around the critical temperature, at which point it approaches infinity. Changes in the absolute viscosity, although less severe in the transitional region, are no less dramatic over the range of temperatures plotted, 
decreasing more than one order of magnitude and approaching values typically found in gases. As a result of these changes the fluid behavior becomes similar to that of a relatively dense, non-polar gas. Additionally, the solvating properties of supercritical water are such that normally immiscible non-polar organics (e.g., alkanes) as well as gases (e.g., $\mathrm{CO}_{2}$, $\mathrm{O}_{2}, \mathrm{~N}_{2}$ ) become highly soluble.

The high solvating capabilities of supercritical water and its high mass diffusion rates consequently make it an ideal medium in which to carry out oxidative reactions. In fact, it has long been observed that, despite the high quenching capacity of water, flaming combustion can often occur [2]. This phenomenon has recently been studied in the context of designing next generation SCWO reactors $[3,4]$ involving internally heated systems with hydrothermal flames as the primary heat source. Waste streams with as little as $10 \%$ hydrocarbon content could be self sustaining. These designs would possibly eliminate many of the issues surrounding corrosion and fouling of the vessel walls, which in current systems, serve as the primary heat transfer surface.

It is interesting to note the behavior of two key parameters that govern buoyant behavior and thermal transport in thermal-fluid systems; the thermal expansion coefficient, $\beta$, and the thermal diffusivity, $\alpha$. These are plotted in Figure 2 for water at a constant pressure of $250 \mathrm{~atm}$ for temperatures up to $700{ }^{\circ} \mathrm{C}$.

The thermal expansion coefficient is given as:

$$
\beta=-\frac{1}{\rho} \cdot\left(\frac{\partial \rho}{\partial T}\right)_{P}
$$

As noted in the previous plot the density of water undergoes a sudden change as the temperature passes through the critical point resulting in a rapid increase in, $\beta$. In normal gravity, as the fluid passes through the transitional zone, the sudden increase in the thermal expansion coefficient will cause large buoyant flows due to the presence of the temperature induced density gradient.

As the fluid undergoes a rapid expansion through the transition zone it is interesting to note that there is an equally sharp decrease in, $\alpha$; which is given as:

$$
\alpha=\frac{k}{\rho \cdot C_{p}}
$$

This parameter is an indication of the fluid's ability to respond to changes in distributions of thermal energy. Therefore, as the fluid system becomes increasingly sluggish in its thermal response, temperature gradients begin to develop that will provide a strong driving

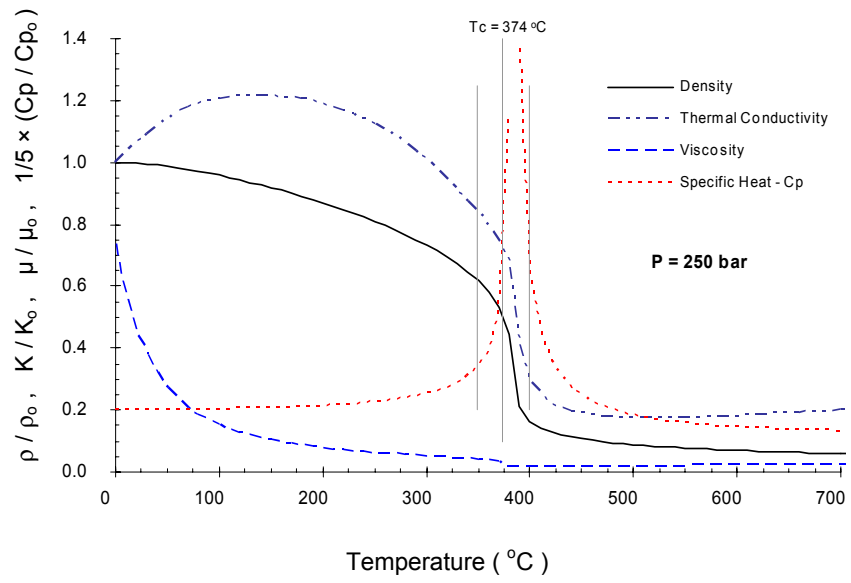

Figure 1 Normalized properties of water at a pressure of 250 bar plotted as a function of temperature, where the reference state is taken at $0^{\circ} \mathrm{C}[1]$.

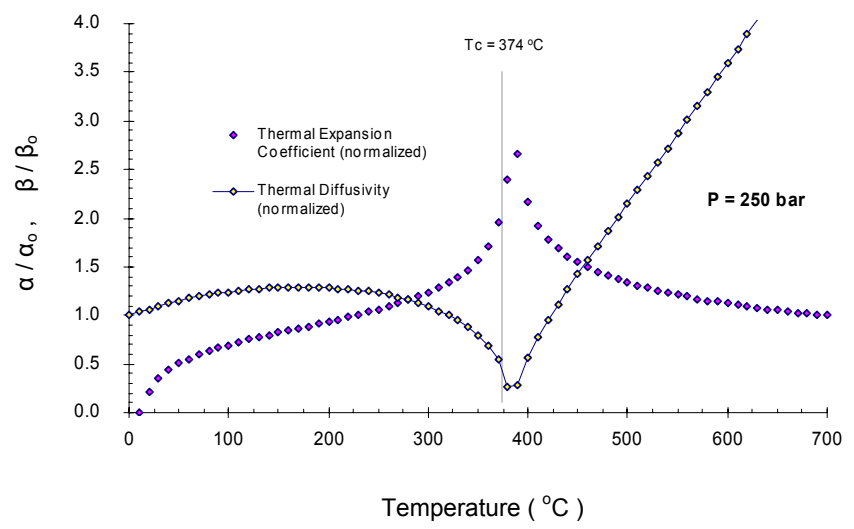

Figure 2 Normalized thermal parameters of water at a pressure of 250 bar plotted as a function of temperature, where the reference state is taken at $0^{\circ} \mathrm{C}[1]$.

potential for buoyant flow. Consequently, a substantial internal mixing potential naturally exists in 1-g by virtue of the sudden changes in thermal properties as the fluid transitions from sub-critical to supercritical.

Most SCWO applications avoid operating conditions near this transitional region due to the sudden changes in thermophysical properties and the resultant difficulty in maintaining system stability. Nonetheless, as the fluid progresses through this transitional region to reach the operating conditions the end state could be significantly altered in $0-\mathrm{g}$.

It is clear that buoyant phenomena will need to be thoroughly understood in order to properly design space/extraterrestrial SCWO systems to ensure that the absence of gravity (or reduction in gravity) does not become problematic. In $0-g$ applications it may be essential that mechanical mixing of the reactor fluid be provided in order to protect against excessive local temperatures, spontaneous flaming, or poor reactant 
distribution. Additionally, in future systems that are designed to operate with some level of flaming combustion, a detailed understanding of the flame dynamics and structure in $0-\mathrm{g}$ will be necessary. As such, test results reported in this paper describe the initial attempts at understanding the hydrodynamics, thermal transport, and reactant mixing in $0-\mathrm{g}$ environments.

\section{SCWO ZERO GRAVITY TEST FACILITY}

Earlier work [5] described a 1-g SCWO Test Facility that was used as a precursor for the design and build-up of the current SCWO Zero Gravity Test Facility. This facility is the product of a series of modifications made to the earlier 1-g facility to allow it to sustain the high landing loads resulting from the $5.2 \mathrm{~s}$ freefall in NASA Glenn's Zero Gravity Facility. Also, since the drop tower is evacuated prior to each drop, an air enclosure was necessary to house some of the normally air-cooled electronics (e.g., on-board Raman spectrometer CPU, power supplies, and laser). A schematic of the mechanical layout, a solid model sketch of the major components, and a picture showing the hardware integrated into one of the ZGF's drop vehicles are presented in the Appendix (i.e,. Figure A$1, \mathrm{~A}-2$, and $\mathrm{A}-3$ ).

A discussion of the major sub-systems of the SCWO Zero Gravity Test Facility follows:

REACTOR VESSEL - The SCWO reactor is constructed from alloy C-276 (commercially available as Hastelloy C-276) with an internal volume of $480 \mathrm{cc}$. The vessel has been ASME certified for a Maximum Allowable Working Pressure (MAWP) of $40.7 \mathrm{MPa}$ at $550^{\circ} \mathrm{C}$. It is a custom design, and uses a bolted split-cap enclosure system with a high temperature grafoil gasket. The head is clamped to the cylinder by a stainless steel ring which has been split into two sections. These sections slide into place from the sides without interfering with any fittings attached to the head. This provides sufficient pressure on the grafoil gasket and provides a very reliable seal at high temperatures and pressures. This design eliminates the possibility of operator error provided the gasket is seated correctly and the bolts are properly torqued.

There are a total of four access ports into the reactor; three on the bottom and one located on the top. Two of the ports on the bottom are fluid lines and the third bottom port serves as an access port for a thermocouple rake. The fourth port, located at the center of the top of the reactor, serves as an access port for a 3/8 inch diameter Raman Probe. This is a specially manufactured fitting which interfaces with the reactor using a $3 / 4$ " medium pressure connection. The interface with the probe body is designed with a "soft" grafoil crush seal to allow vertical positioning within the reactor vessel. A picture of the reactor vessel along
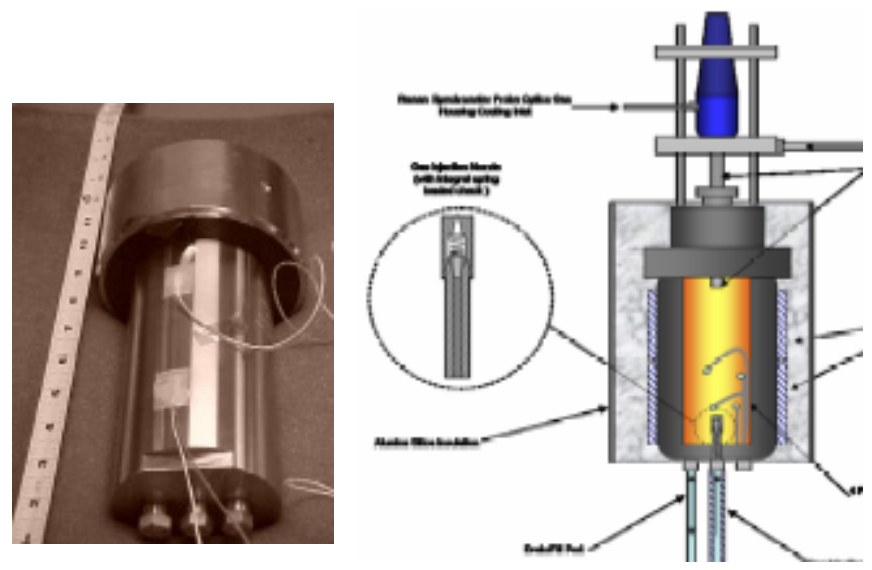

Figure 3 Reactor vessel along with schematic of reactor vessel cross-section showing location of oxidizer injector, thermocouple rake (in radial configuration), and Raman Spectrometer Probe.

with a schematic illustrating placement of the Raman Probe and thermocouple rake is presented in Figure 3.

HEATING ELELMENTS - The reactor heater consists of a pair of 1,500 watt band heating elements that can reach a maximum set-point temperature of $800^{\circ} \mathrm{C}$. The heaters are "on-off" controlled by an internal thermocouple located on the center-line of the reactor vessel. Each band heater is instrumented with a Type- $\mathrm{K}$ thermocouple and the top thermocouple is wired to a panel meter for health and monitoring.

A second heating element is used to pre-heat the oxidizer line between the reactor and the gas injection valve to ensure that the oxidizer, when injected into the charged reactor, is at a temperature close to the reactor fluid temperature. This is accomplished with a 300 watt $1 / 2 "$ × 6' tape heater wrapped around the 2 ' length of oxidizer injection tube. This heater is controlled by a thermocouple in direct contact with the outside of the tube.

ON BOARD GAS SUPPLY - The ZGF testing was designed to allow for the start of the SCWO reaction to be controlled by the injection of the oxidizer, which in this case was standard air (i.e., $21 \% \mathrm{O}_{2}$ balance $\mathrm{N}_{2}$ ). A pressurized "on-board" air supply was required and consisted of two Department of Transportation (DOT) certified gas cylinder; one with a $500 \mathrm{ml}$ capacity and the second with a $300 \mathrm{ml}$ capacity and both rated for a MAWP of $34.5 \mathrm{MPa}$. At the conclusion of each test a gas sample was drawn from the reactor vessel and this was stored in an on-board sample bottle with a $150 \mathrm{ml}$ capacity and a MAWP of $12.4 \mathrm{MPa}$.

OXIDIZER INJECTION - The oxidizer injector is designed to introduce the oxidant into the reactor by either a radial discharge or an axial discharge. This design flexibility allows for the initial reaction to proceed as either a partially pre-mixed reaction (i.e, radial injection) or a diffusion-limited reaction (i.e., axial jet injection). 
Although all testing reported in this work uses the axial jet configuration the radial injector could have been used by simply interchanging injectors. A schematic of these two injector designs is presented in Figure 4.

ACTIVE COOLING - There are three shell and tube heat exchangers in the system, each rated at a MAWP of $34.5 \mathrm{MPa}$ at $540^{\circ} \mathrm{C}$. These are used for cooling temperature sensitive components as well as the reactor fluids immediately after each test. Each heat exchanger has approximately 38 ' of coiled $1 / 4$ " or $3 / 8$ " OD tubing (depending on the model) inside a high pressure shell.

ACCUMULATOR - The SCWO Zero Gravity Test Facility is unique in that it can operate as either a constant volume reactor (i.e, batch reactor) or a constant pressure reactor (i.e, continuous flow reactor). In the constant pressure operational mode, compensation for fluid expansion, during heat up and reaction phases, is accomplished by a piston accumulator, which is rated at a MAWP of $34.5 \mathrm{MPa}$.

ELECTRONICS AIR ENCLOSURE - In order to avoid exposure of sensitive electronic equipment to the high vacuum environment of the drop tower, a special enclosure is used. This is made from a 12" O.D. 6061-T6 aluminum extrusion with a $1 / 2$ " wall thickness and is approximately 22" inches in overall length. All electronics are mounted to a plate inside the extrusion. There are two plates on either end of the extrusion that interface with an O-ring seal and are secured by four steel tie rods.

SCIENCE DIAGNOSTICS - Apart from a wide assortment of pressure and temperature diagnostics used primarily for health and status, the science diagnostics consist of a Raman Spectrometer and four internal thermocouples arranged either axially, along the reactor's center axis or radially, in pairs at two predetermined heights. Testing reported in this work only used the axial configuration.

In addition to the recorded data, a post-test analysis of the reactor contents is performed. The reactor fluid is immediately isolated, by activation of a solenoid valve, and a sample is then drawn from the reactor vessel. A chromatographic analysis of both the gas and liquid samples is performed to obtain post-test concentrations of the reactants.

The Raman Spectroscopy System is a custom made diagnostic tool developed specifically for SCWO. It is designed to perform in-situ quantitative analysis of the chemical reactions taking place in the SCWO reactor. It has a Hastelloy insertion tube with a sapphire window to permit passing of the excitation laser and the returned Raman scattering. The probe is rated to withstand $500{ }^{\circ} \mathrm{C}$ and a MAWP of $34.5 \mathrm{MPa}$. The spectrometer, housed in the electronics air enclosure,

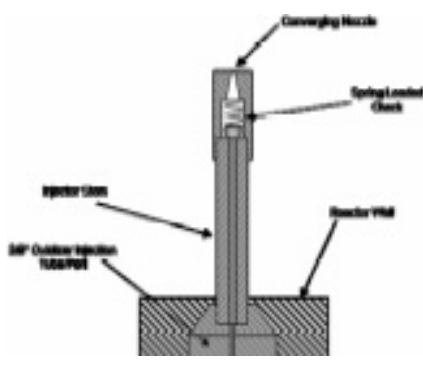

(a)

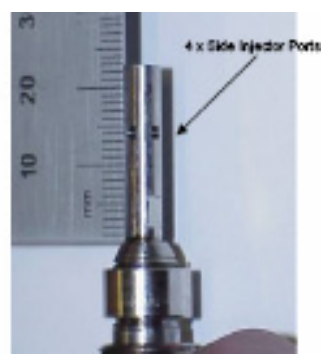

(b)
Figure 4 Oxidant injector configurations; (a) axial jet flow and (b) radial injection.

is equipped with a thermo-electrically cooled $1024 \times 64$ array detector. The Raman scattering is stimulated by a $100 \mathrm{~mW} 532 \mathrm{~nm}$ diode pumped YAG laser. A schematic of the system and the actual hardware is presented in the Appendix (Figures A-4 and A-5).

\section{SCWO - ZERO GRAVITY TEST PROCEDURES}

General test procedures include the following breakdown of activities:

NITROGEN PURGE - A nitrogen purge is performed prior to each test to eliminate previous experiment effluent and un-reacted fuels that may have remained in the system after prior test samples were drawn. This purge gas will flow through all wetted components exposed to the previous experiment.

OXIDIZER PRE-CHARGE - The oxidizer (i.e., standard air for all tests) reservoirs are filled to the appropriate pressure to ensure adequate on-board supply for each test. Once both oxidizer cylinders are at the predetermined pressure the reservoirs are isolated from the rest of the system until the test commences.

ReActor Fill AND PREssurizATION - After the lines and the reactor vessel have been purged with $N_{2}$ the reactor is filled with the aqueous test mixture. A vacuum is drawn on the entire line from the fill port to the reactor vessel to ensure no air or $\mathrm{N}_{2}$ remains in the system. An oversized syringe, filled with the test mixture, is then attached to the fill port fitting and slowly metered into the reactor. After filling the reactor vessel and line, from the reactor to the accumulator, the fluid is pressurized by pressurizing the back side of the accumulator with $\mathrm{N}_{2}$ to the target test pressure.

REACTOR HEATING - Once the SCWO ZGF Test Facility has been lifted to the top of the ZGF drop tower a 220V electrical umbilical is attached to the test rig and reactor heating commences. As the reactor increases in temperature, the fluid/vapor mixture in the vessel expands, causing the piston in the accumulator to 
gradually move to compensate for the expansion. This allows a constant pressure to be maintained in the reactor during heat-up and testing.

OXIDIZER INJECTION - A prescribed pressure differential between the initial pressure in the oxidizer bottle and the bottle's "post injection" pressure is used to determine the quantity of oxidizer that is to be injected into the reactor for the test. An algorithm is used to automatically close the gas injection valve once the oxidizer bottle pressure drops below the target pressure.

The experiment is ready to start once the targeted supercritical conditions have been reached in the reactor. The test begins by opening the high pressure selection valve (which allows selection of which oxidizer bottle to draw from) and the gas injection valve. This allows the oxidizer to flow into the SCWO reactor through the injector nozzle (refer to Figure 4), which is centered in the bottom of the reactor. The SCWO reaction starts as soon as the oxidizer is introduced into the reactor. The SCWO ZGF Test Facility can then be released for its 5.2 second freefall at any point in the test sequence depending on the objectives of the test.

\section{RESULTS AND DISCUSSION}

TEST RESULTS - Two successful tests were performed in the ZGF using an aqueous mixture of $10 \%$ methanol (i.e., $\mathrm{CH}_{3} \mathrm{OH}$ ). In Test 1 a relatively slow injection (i.e., $45 \mathrm{~cm} / \mathrm{s}$ ) of the oxidizer was performed and in Test 2 a faster injection (i.e,. $200 \mathrm{~cm} / \mathrm{s}$ ) was performed. In each case, just prior to the onset of $0-g$, the oxidizer was injected into the reactor in order to clear the line of any reactor fluid which may have migrated past the injector seal into the oxidizer line. This allows a 1-g oxidation reaction to occur serving as a baseline for comparison with the subsequent $0-g$ reaction.

The sequence of events for both tests can be partitioned into five test phases; (i) 1-g oxidizer injection, (ii) 1-g SCWO reaction, (iii) 1-g temperature stabilization, (iv) 0-g oxidizer injection, and (v) $0-g$ SCWO reaction. Each test was initiated once the reactor fluid temperature was stabilized at $450^{\circ} \mathrm{C}$ with the reactor pressure held constant at a nominal $250 \mathrm{~atm}$. Both tests were operated in the constant pressure mode and pressures were successfully maintained to within $1.2 \%$ for Test 1 and to within $5.6 \%$ for Test 2. A pressure profile through all test phases is presented in the Appendix as Figures A-6, for Test 1, and A-7, for Test 2. These plots show both the reactor pressure and the oxidizer reservoir pressure. The rate of injection was controlled with a metering valve which was set prior to each test and the amount of oxidizer that was injected was controlled by setting a target pressure for the oxidizer reservoir.
The primary diagnostic for these tests were the temperature traces provided by the internal thermocouples. Although Raman spectroscopy measurements were successfully made for a series of earlier 1-g tests these were not available for the two ZGF tests due to difficulties with the on-board power supply. As stated earlier, the axial jet flow configuration for the oxidizer injection was used and the internal thermocouples, labeled from bottom (i.e., TC-A) to top (i.e., TC-D), were aligned along the vertical axis of symmetry of the reactor. These were spaced $3.8 \mathrm{~cm}$ apart with the first thermocouple located $1.3 \mathrm{~cm}$ from the exit plane of the oxidizer injector.

Transient internal reactor temperatures are presented in Figure 5 for both tests. Figures 5(a), and 5(b) show temperature profiles from Test 1 , which was the "slow" oxidizer injection with an oxidizer exit velocity of $45 \mathrm{~cm} / \mathrm{s}$. The $1-\mathrm{g}$ oxidation reaction is presented in Figure 5(a) and is characterized by a rather narrow temperature range. The temperature spread is less than $6{ }^{\circ} \mathrm{C}$ at the point of peak temperature between the upper three thermocouples (i.e., TC-B, TC-C, TC-D) and the average rate of temperature rise is approximately $2.1^{\circ} \mathrm{C} / \mathrm{s}$. Since thermocouple TC-A is located near the exit plane of the injector this is dominated by the oxidizer temperature and consequently lags behind the other TC's. These temperature profiles are compared with the $0-g$ reaction shown in Figure 5(b) where the temperature spread between the upper three thermocouples suddenly grows, from $6^{\circ} \mathrm{C}$ in $1-\mathrm{g}$, to over four times that by the end of the $5.2 \mathrm{~s} 0-\mathrm{g}$ period (i.e., $17^{\circ} \mathrm{C}$ between TC-C and TC-D). Additionally, the average rate of temperature rise in $0-\mathrm{g}$ is $5.1^{\circ} \mathrm{C} / \mathrm{s}$, over twice that observed in 1-g. It is apparent that sudden changes in the hydrodynamic flow field occur with the transition to $0-g$. Even though there is probably some residual convective flow well into the 5.2 second $0-\mathrm{g}$ period it is interesting that there is such a significant localized temperature effect associated with the absence of any buoyantly induced convective transport of mass and thermal energy from the reaction sites.

The results from Test 2 , having nearly identical test conditions as that of Test 1 , show the effects of suppressed mixing in $0-g$ from a slightly different perspective. In this case the oxidizer injection velocity of $200 \mathrm{~cm} / \mathrm{s}$ was nearly 4.5 times the injection velocity of Test 1. As a result, the injection period was considerably shorter in both $1-\mathrm{g}$ and $0-\mathrm{g}$. In this case the oxidizer injection was terminated well before the 0 $\mathrm{g}$ period began and the temperature peak had already been reached. This indicates that the oxidation reaction had come to completion just prior to release. At this point, once the fuel has been consumed, the temperature will drop as thermal equilibrium is reached with the bulk fluid and reactor walls. It is observed that in 0 -g the rate of cooling was nearly half that observed in 1-g for the same time period immediately following 
peak temperature conditions (i.e., $1.4^{\circ} \mathrm{C} / \mathrm{s}$ compared to $2.4^{\circ} \mathrm{C} / \mathrm{s}$ ). Additionally, during cooling in $0-\mathrm{g}$, the temperature difference between TC-A (bottom) and the upper TC's persists as is shown in Figure 5(d). This contrasts with the cooling profile in 1-g where buoyant mixing quickly diminishes any temperature spread, as evidenced by the convergence of all TC traces following the peak temperature rise in Figure 5(c).

A sample of both the liquid reactor contents and the gas/vapor mixture, vented from the reactor, was obtained immediately following the $0-\mathrm{g}$ portion of Test 2. Results from this analysis showed that approximately $98 \%$ of the methanol was oxidized during the combined $1-\mathrm{g}$ and $0-\mathrm{g}$ reactions.

ANALYSIS - The observed effect on internal temperature profiles is illustrated by two transient numerical analyses; the first simply looking at a combined buoyant/forced flow field of a non-reacting axisymmetric jet and the second looking at a diffusionlimited reacting jet. In previous work [5], two modes of operation were identified; i.e., a non-premixed (i.e., "diffusion limited") mode and a premixed mode. In the diffusion-limited mode reactants are kept separate until onset of oxidation, which is limited by diffusion of the reactants to the reaction zone (i.e., assuming reaction rates are sufficiently high, so as not to be the limiting rate). In the premixed mode, reactants are well mixed at the molecular level prior to oxidation.

The present discussion is limited to the diffusion-limited mode. As in the experimental results, discussed in the preceding section, consider the case where one of the primary reactants (e.g., oxidizer), denoted by subscript, $i$, is injected into the reactor containing supercritical water and the other reactant (e.g., the waste) denoted by subscript, w. Assume also that the constituent species are in single phase. Define a mixing variable, $Y$, which depends upon the mass fractions of the two primary reactants, $Y_{i}$, and , $Y_{W}$, as follows:

$$
\mathrm{Y}=\left[-\frac{\mathrm{Y}_{\mathrm{w}}}{\mathrm{W}_{\mathrm{w}} v_{\mathrm{w}}}+\frac{\mathrm{Y}_{\mathrm{i}}}{\mathrm{W}_{\mathrm{i}} v_{\mathrm{i}}}\right] \cdot \frac{\mathrm{W}_{\mathrm{i}} v_{\mathrm{i}}}{\mathrm{Y}_{\mathrm{i}, 0}}
$$

Where, $v_{\mathrm{i}}$ and $v_{\mathrm{w}}$, are the stoichiometric coefficients for species "i" and " $w$ ", respectively, and the molecular weights are given as $W_{i}$ and $W_{w}$.

Combining the species transport equations for, $Y_{i}$, and, $Y_{W}$, yields the following equation for, $Y$, in stream function coordinates:

$$
\frac{\partial Y}{\partial x}=\frac{1}{\Psi} \cdot \frac{\partial}{\partial \Psi} \cdot\left(\frac{u \cdot r^{2}}{\Psi} \frac{\partial Y}{\partial \Psi}\right)
$$

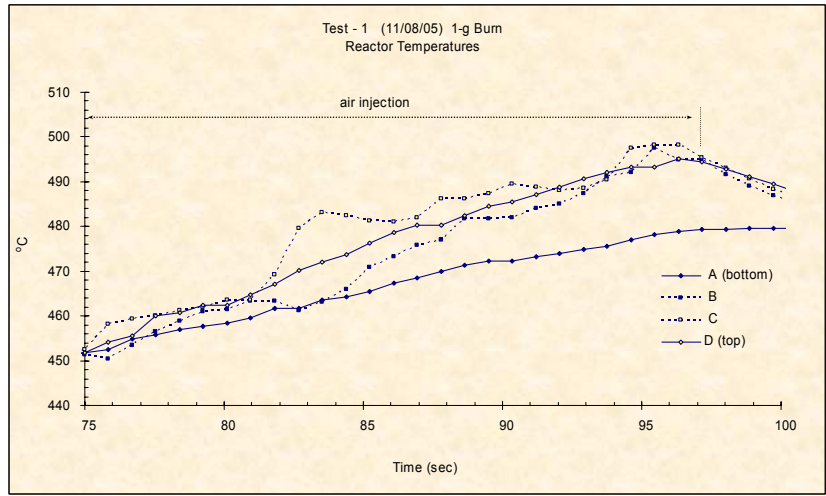

(a)

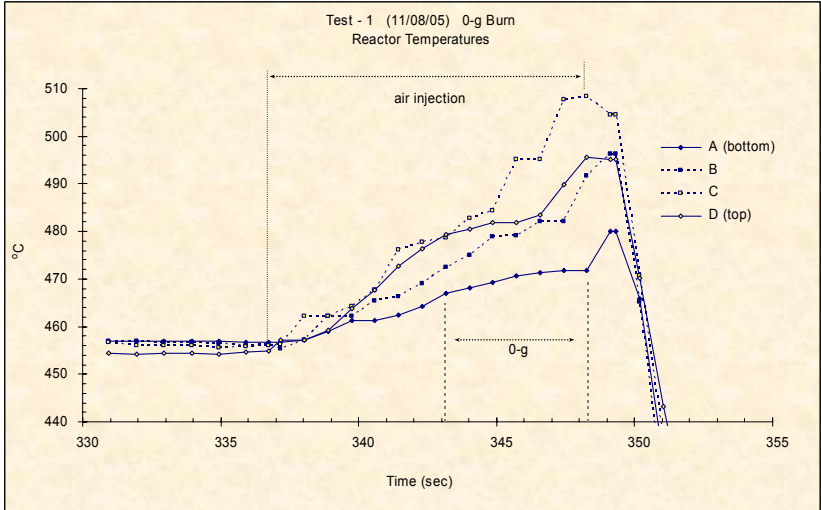

(b)

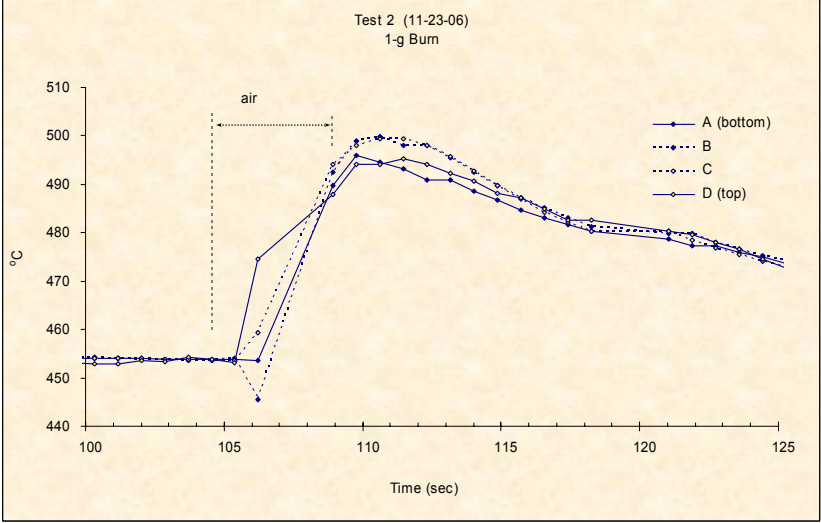

(c)

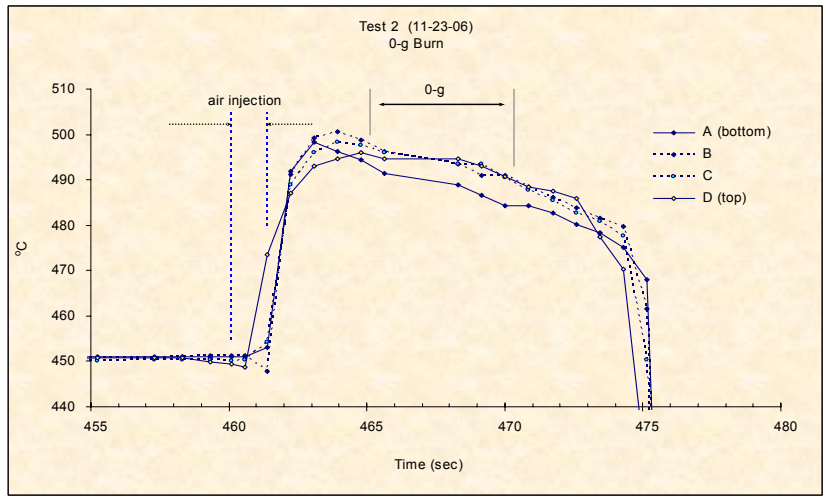

(d)

Figure 5 Internal axial transient temperature profile immediately following oxidizer injection for Test 1, slow injection at $45 \mathrm{~cm} / \mathrm{s}$ in 1-g (a) and $0-\mathrm{g}$ (b) and Test 2, a rapid injection at $200 \mathrm{~cm} / \mathrm{s}$ in $1-\mathrm{g}$ (c) and $0-\mathrm{g}$ (d); pressure held constant at a nominal $250 \mathrm{~atm}$ throughout each test. 
where: $\quad u \cdot r=\Psi \frac{\partial \Psi}{\partial r} \quad$ and $\quad-v \cdot r=\frac{\Psi}{P e} \frac{\partial \Psi}{\partial x}$

In the above, $u$ and $v$ are the axial and radial velocity components normalized by the injection velocity, $u_{0}$. The axial and radial coordinates are normalized by the radius of the injector port, $\mathrm{b}$. The quantity, $\mathrm{Pe}$, is the Peclet number given by:

$$
P e=\frac{u_{0} b}{D}
$$

where, $D$ is the molecular diffusion coefficient assumed here to be constant and the same for all species.

Gravity directly impacts the distribution of, $Y$, by affecting the velocity field. The importance of gravity may be assessed by considering the momentum equation. If the Reynolds number, Re, of the injected flow is much greater than 1 , as is the case here, the proper parameter to consider is the Richardson number, $\mathrm{Ri}$, which is defined as the ratio of buoyancy force to inertia force:

$$
\begin{aligned}
& \mathrm{Ri}=\left(\frac{\Delta \rho}{\rho}\right) \frac{\mathrm{g} \cdot \mathrm{I}}{\mathrm{u}_{0}^{2}} \\
& \mathrm{I}=\text { characteristic length scale }
\end{aligned}
$$

If $\mathrm{Ri} \gg 1$, then the axial velocity varies as, $\mathrm{u} \propto \sqrt{\mathrm{g} \cdot \mathrm{x}}$, where, $\mathrm{x}$, is the distance from the injection point. A consequence of this acceleration is enhanced entrainment of the surrounding fluid toward the axis of injection. This tends to squeeze the streamlines of the flow together which, in turn, enhances the diffusion of mass. Generally, these two effects tend to decrease the spatial extent of the reaction region compared to the zero gravity case, i.e., the "flame" is smaller.

Changes in the layout of the streamlines may have bearing also on the deposition of salts that may be formed. For $\mathrm{Ri}>>1$, the streamlines tend to converge toward the injection axis so that particulates that may be formed at the reaction zone would tend to travel out from near the tip of the reaction region. However, their path would also depend upon agglomeration characteristics, residence time in the reaction region and their relative density compared to the surrounding medium. For the zero gravity case on the other hand, there is no convergence of the streamlines due to buoyancy and the particulates would tend to follow the dividing streamline i.e., outward in this case.

Numerical simulations to illustrate these assertions were carried out. First, the flow field behavior for normal and zero gravity are illustrated without the complications arising from chemical reaction by simply simulating the injection of a hotter fluid into the supercritical water reactor. Figure 6 shows the velocity vectors and temperature map for a supercritical water jet, at $800 \mathrm{~K}$, injected into a reactor filled with supercritical water at $700 \mathrm{~K}$ for both normal gravity and zero gravity cases. The computation includes time dependence and the figure depicts conditions at the same time following injection. The entrainment and higher velocities near the central axis in normal gravity are clearly seen. The zone of temperature spread is significantly narrower in normal gravity because of buoyancy. The zero gravity simulation shows the impact of lack of buoyant acceleration and a radial spread of the heated jet results. It is only in the immediate neighborhood of the injection, where inertial forces dominate, that the normal gravity and zero gravity behavior are similar.

Similar results are obtained from the reacting case where supercritical water in the reactor contains the waste material and the oxidant is injected. Figure 7 shows the concentration of the injected species and temperature maps with superimposed velocity vectors in the extended neighborhood of the injection location region of the reactor. The temperature begins to increase after injection as the energy of the chemical reaction is released. The reaction occurs at the interface between the reactants. As before, the increased radial spread in the temperature field for the zero gravity case is evident. Also of note is that the temperatures in the downstream region of the computational domain are hotter for the zero gravity case presumably due to the decrease in energy convection out of the domain because of the reduced velocities. It is also worth noting that the injected species (i.e., oxidizer) does not show the same degree of radial spread as observed in the temperature profiles. This is, in part, because the species diffusion rates are much smaller than the temperature diffusion rate.

\section{CONCLUSION}

A SCWO Zero Gravity Test Facility has been built which can be used in NASA Glenn Research Center's 5.2 second drop tower. This facility comprises a $480 \mathrm{ml}$ high pressure reactor vessel along with supporting equipment that allows for a wide range of SCWO reactions to be studied in either a constant pressure mode (i.e, analog for continuous flow reactors) or a constant volume mode (ie., a batch reactor).

Early results from two tests using an aqueous mixture of $10 \%$ methanol (i.e., $\mathrm{CH}_{3} \mathrm{OH}$ ), oxidized by air injected into the supercritical mixture, are reported for both 1-g and $0-g$ environments. The two tests were designed to demonstrate the effects on the temperature field when buoyant induced mixing is removed. Test 1 injected the oxidizer at a velocity of $45 \mathrm{~cm} / \mathrm{s}$ and Test 2 injected 

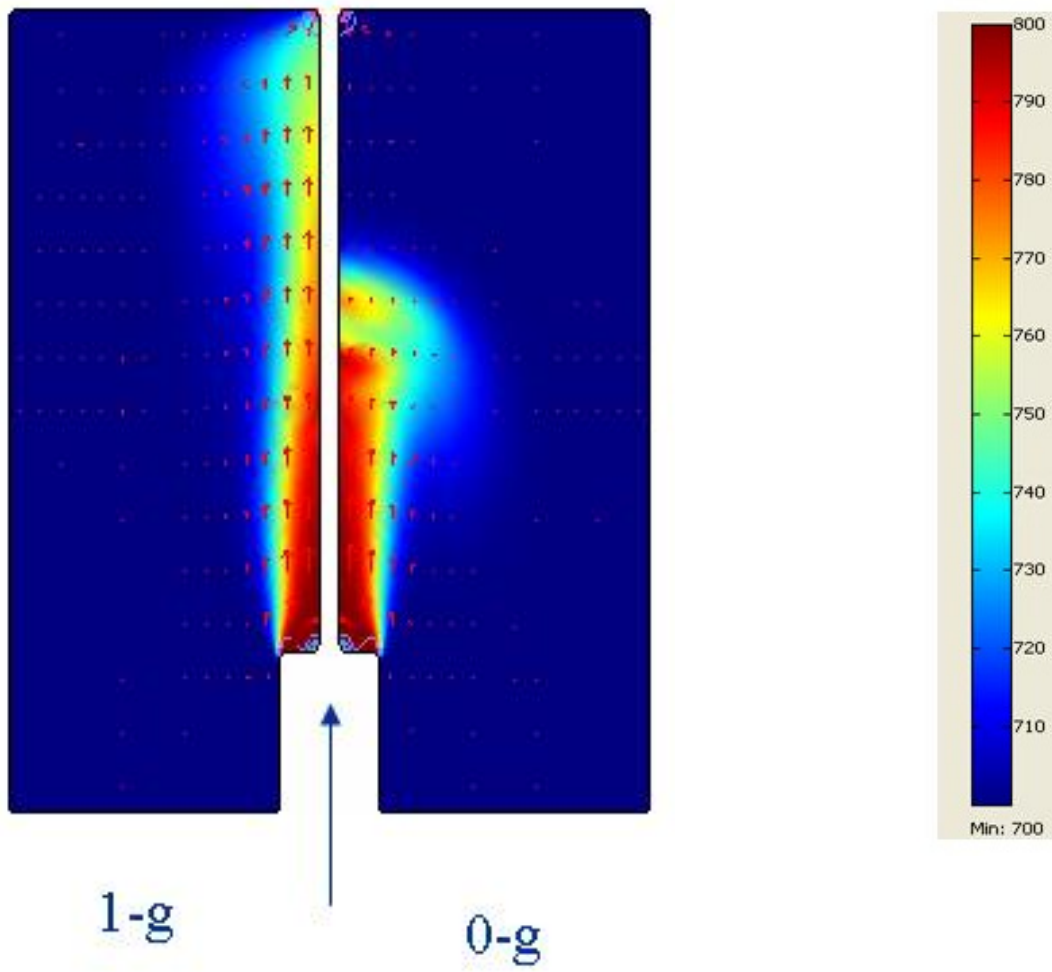

Figure 6 Illustration of buoyancy effects showing non-reacting axysymmetric jet of heated fluid injected into body of cooler fluid.

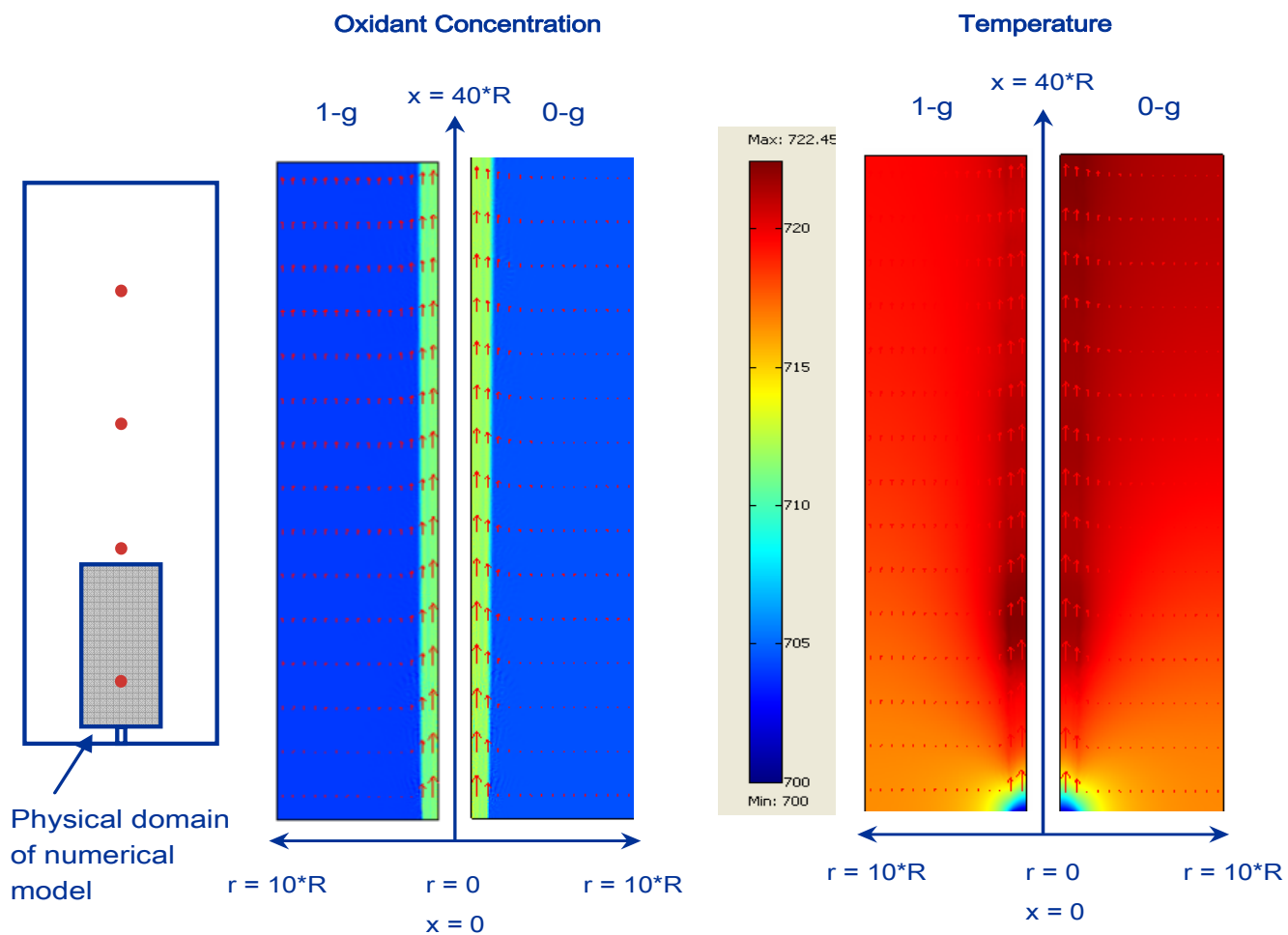

Figure 7 Numerical results of reacting axisymmetric jet of oxidizer injected into supercritical water and methanol mixture. 
the oxidizer at a velocity of $200 \mathrm{~cm} / \mathrm{s}$. Comparisons are made between internal thermocouples placed in the reaction zone and results show significant differences in temperature uniformity and rate of temperature change both during and immediately following reaction as a result of the absence of buoyant forces.

A numerical analysis of the test configuration was performed to illustrate the effects of the absence of gravity and to identify the important mixing parameters. A non-reacting flow and a reacting flow configuration were presented and results showed significant differences in reactant distribution, flow field, and temperature profile between $0-g$ and $1-g$ environments.

Through testing and analysis it has been shown that gravitational influences on reactant mixing and thermal transport will need to be clearly understood in order to properly apply SCWO technology in space and/or extraterrestrial applications. SCWO reactors designed to operate in 1-g environments will behave differently in $0-g$ or reduced gravity environments because of the absence of buoyant mixing. The operating efficiency of a SCWO system, driven largely by reactant distribution and thermal transport, will depend to a much greater extent on the method of reactant injection, the method of reactant heating, the chosen process mode (i.e., batch or continuous flow), as well as the reactor geometry. Results from these early tests have shown the development of localized temperature gradients that are not evidenced in 1-g. Higher localized temperatures could lead to spontaneous flaming resulting in higher temperatures which, if not properly controlled, may produce unwanted combustion products (e.g., NOx) or damage temperature sensitive reactor components (e.g., reactant injectors, control diagnostics, etc.).

\section{ACKNOWLEDGMENTS}

The authors would like to thank the engineering team at ZIN Technologies for their dedication and commitment in completing the design, build-up and successful integration of the SCWO Zero Gravity Test Facility. It was only through the contributions of that team and in particular through the efforts of Michael Sydenstricker, Roland Lowe, Anthony Bruzas, Gregory
Fedor, and Judy Anthony that this work was made possible.

Additionally the authors would like to thank the personnel of NASA GRC's Zero Gravity Facility, in particular Eric Neumann and Darko Krajl, who made it possible to meet the significant challenges inherent in integrating this unique system into the drop tower.

Finally, the authors would like to acknowledge helpful discussions with John Fisher and Kanapathipi Wignarajah of NASA Ames Research Center who generously shared their insights from earlier work with SCWO.

\section{REFERENCES}

1. NIST Chemistry WebBook, NIST Standard Reference Database Number 69, June 2005.

2. Franck, E.U., "Combustion and Flames in Supercritical Fluids to 2000 bar", Recent Trends in High Pressure Research High Pressure, pages 501-506, Proceding of the $13^{\text {th }}$ AIRAPT International Conference, Oxford \& IBH Publishing Co. Pvt. Ltd., 1992

3. Wellig, B., Transpiring Wall Reactor for Supercritical Water Oxidation, PhD. dissertation submitted to Swiss Federal Institute of Technology Zurich, 2003.

4. Serikawa et. al., "Hydrothermal Flames in Supercritical Water Oxidation: Investigation in a Pilot Scale Continuous Reactor"; Fuel 81 pp 11471159, 2002.

5. Hicks, M.C., Lauver, R.W., Hegde, U.G., Hall, D.G., Sikora, T.J., "Gravity Effects on Premixed and Diffusion Limited Supercritical Water Oxidation", SAE Technical Paper Series No. 2005-01-3036, $35^{\text {th }}$ International Conference on Environmental Systems, Rome, Italy, July $11^{\text {th }}$, ' 05.

\section{CONTACT}

Michael C. Hicks

Microgravity Combustion Science

216-433-6576

mhicks@nasa.gov 
APPENDIX

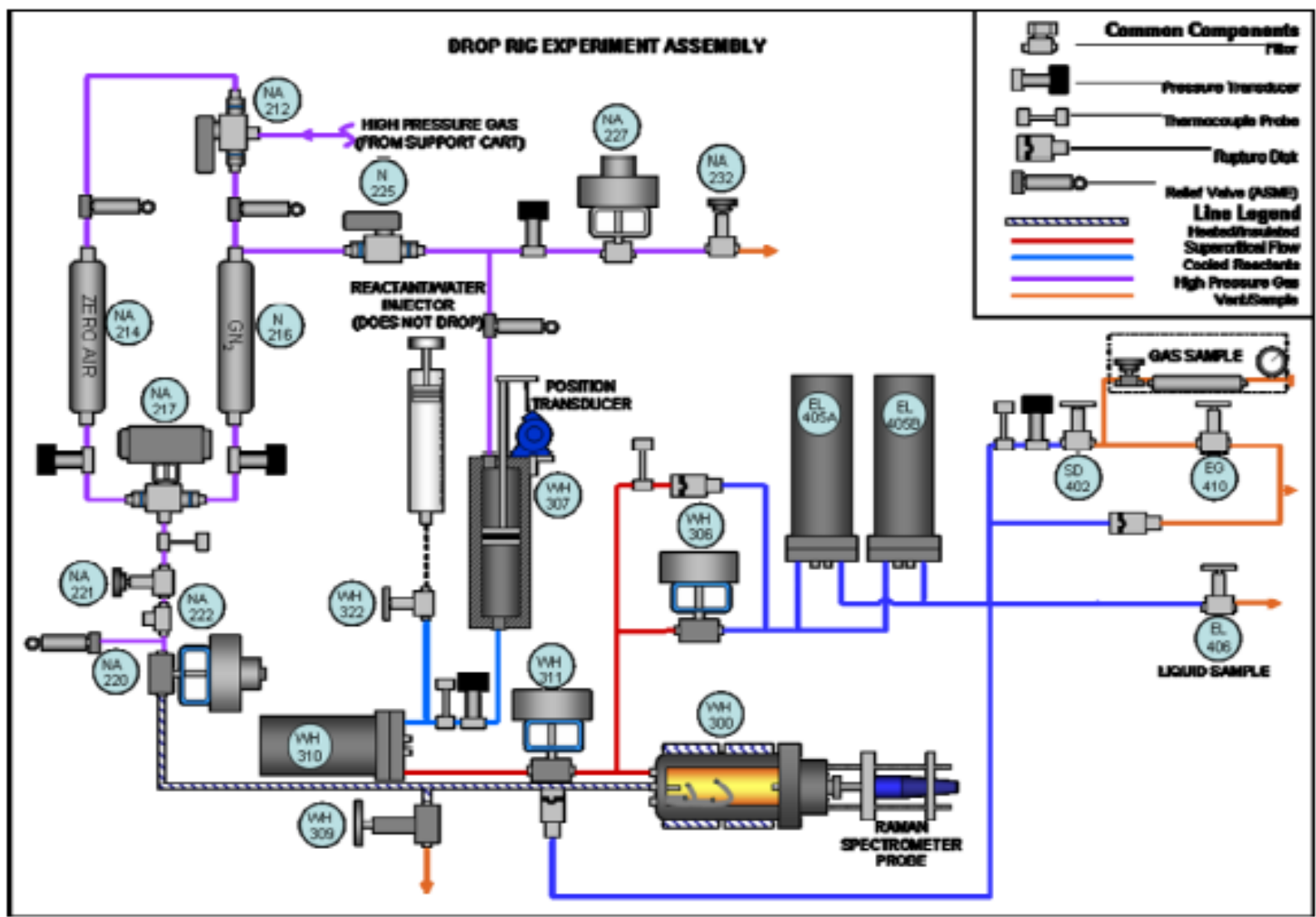

Figure A-1 Mechanical layout of major mechanical components on the SCWO Zero Gravity Test Facility 


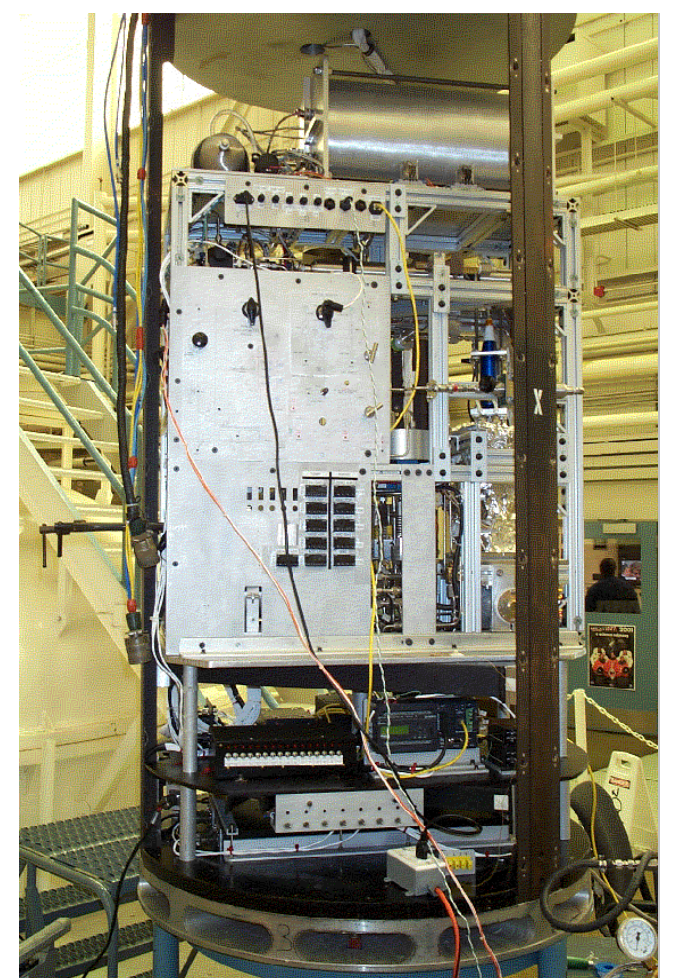

Figure A-2 SCWO Zero Gravity Test Facility (front view) fully integrated in the ZGF drop vehicle just prior to enclosure with outer protective skins.

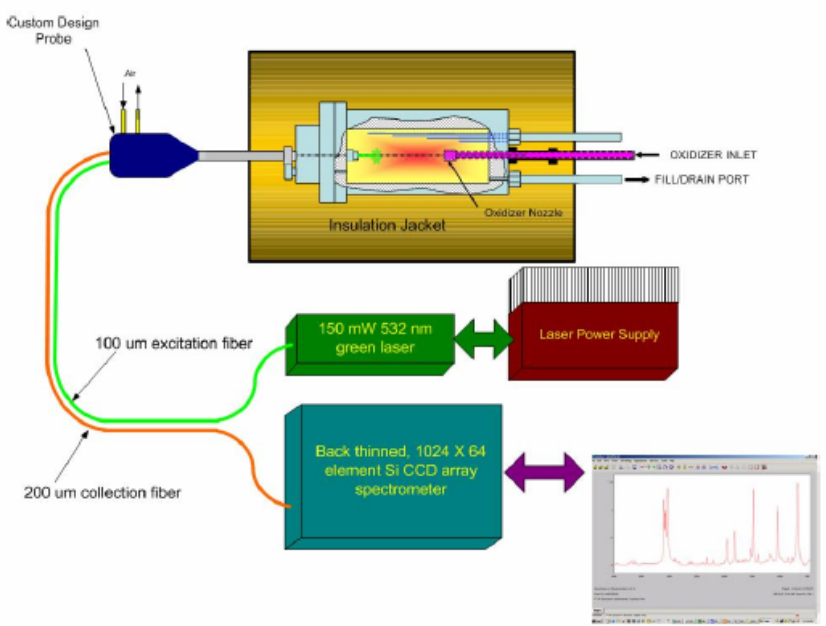

Figure A-4 Schematic of Raman Spectroscopy System
Electronic Air Enclosure

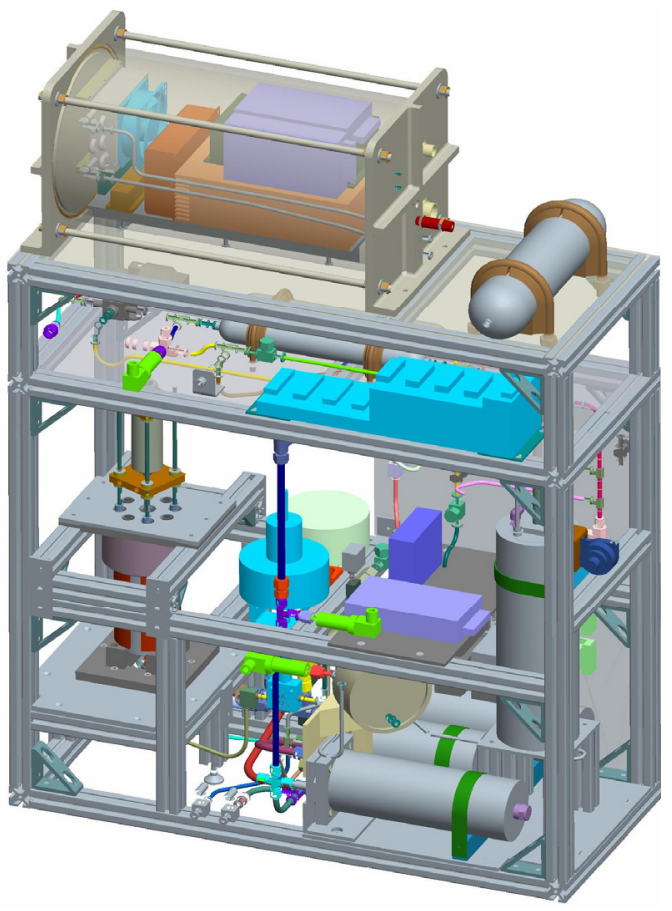

Figure A-3 Solid model of SCWO Zero Gravity Test Facility (rear view) showing back layout of major system components.

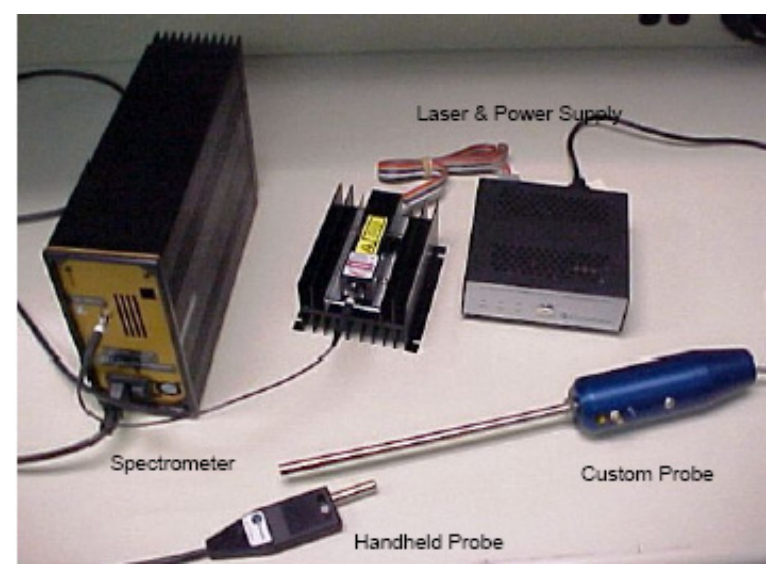

Figure A-5 Raman Spectroscopy System - component parts 
APPENDIX (cont)

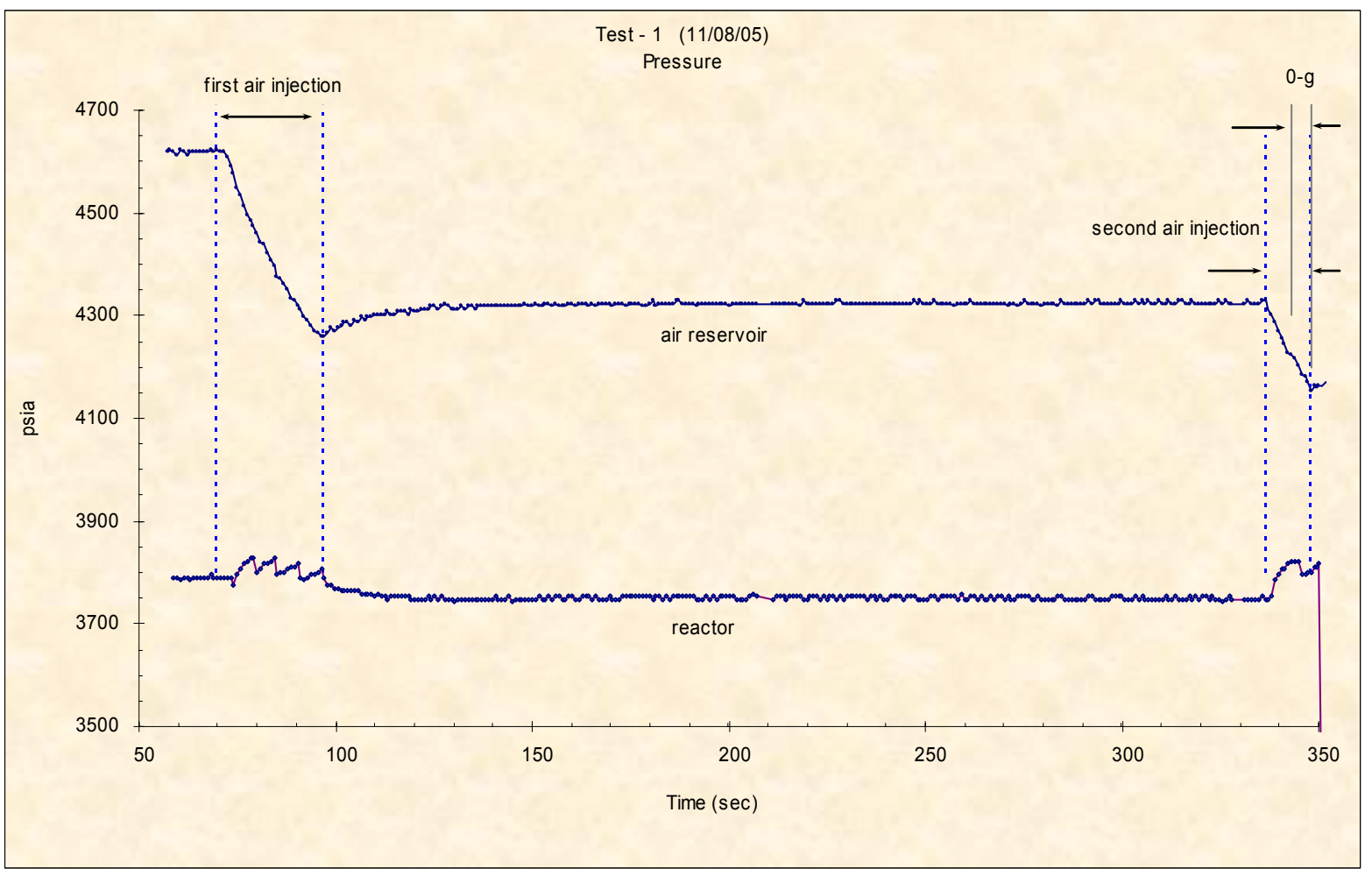

Fig A-6 Test 1 - pressure profile

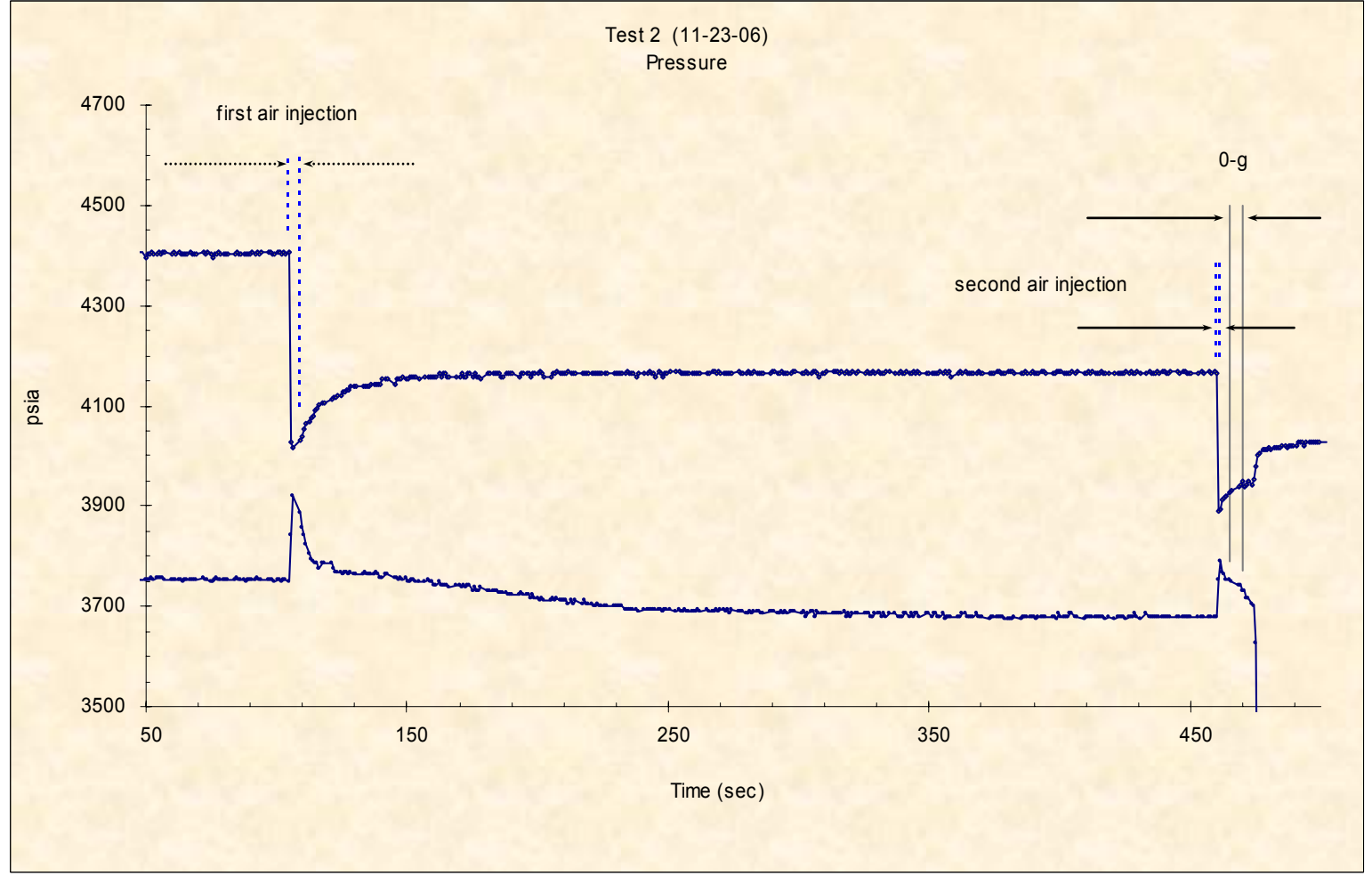

Fig A-7 Test 2 - pressure profile 